\title{
Solution Of Singularly Perturbed Delay Differential Equations Using Liouville Green Transformation
}

\author{
M. Adilaxmi , D.Kumara Swamy* \\ Department of Mathematics, K.L. University, Hyderabad, INDIA \\ Email: madireddyadilaxmi@gmail.com \\ *Department of Mathematics, Indian Institute of Information Technology, Sonepat, India. \\ Email:diddi.k@gmail.com
}

Article History: Received:11 January 2021; Accepted: 27 February 2021; Published online: 5 April 2021

\begin{abstract}
This paper envisages the use of Liouville Green Transformation to find the solution of singularly perturbed delay differential equations. First, using Taylor series, the given singularly perturbed delay differential equation is approximated by an asymptotically equivalent singularly perturbation problem. Then the Liouville Green Transformation is applied to get the solution. The method is demonstrated by implementing several model examples by taking various values for the delay parameter and perturbation parameter.
\end{abstract}

Key words: Singular perturbations, Delay term, Boundary layer

Mathematics Subject Classification: 65L11, 65Q10

\section{Introduction}

A singularly perturbed delay differential equation is an ordinary differential equation in which the highest derivative is multiplied by a small parameter and containing delay term. In recent years, there has been a growing interest in the numerical treatment of such differential equations. This is due to the versatility of such type of differential equations in the mathematical modeling of processes in various application fields, for e.g., the first exit time problem in the modeling of the activation of neuronal variability [9], in the study of bistable devices [2], and variational problems in control theory [5] where they provide the best and in many cases the only realistic simulation of the observed. Stein [11] gave a differential-difference equation model incorporating stochastic effects due to neuron excitation. Lange and Miura [9, 10] gave an asymptotic approach for a class of boundary-value problems for linear second-order differential-difference equations. Kadalbajoo and Sharma [7, 8], presented a numerical approaches to solve singularly perturbed differential-difference equation, which contains negative shift in the either in the derivative term or the function but not in the derivative term. In this paper we describe the use of Liouville Green Transformation to find the solution of singularly perturbed delay differential equations. First, using Taylor series, the given singularly perturbed delay differential equation is approximated by an equivalent singularly perturbation problem. Then the Liouville Green Transformation is applied to get the solution. The method is demonstrated by implementing several model examples by taking various values for the delay parameter and perturbation parameter.

\section{Description of the method}

Consider singularly perturbed singular boundary value problems of the form

$$
L y \equiv \varepsilon y^{\prime \prime}(x)+a(x) y^{\prime}(x-\delta)+b(x) y(x)=0, \quad 0 \leq \mathrm{x} \leq 1
$$

with boundary conditions $\quad y(0)=\alpha,-\delta \leq x \leq 0$

$$
\text { and } y(1)=\beta
$$

where $0<\varepsilon<<1, b(x)$ is bounded continuous function in $(0,1)$ and $\alpha, \beta$ are finite constants. Further, we assume that $a(x) \geq M>0$ throughout the interval $[0,1]$, where $M$ is positive constant. This assumption merely implies that the boundary layer will be in the neighbourhood of $x=0$.

By using Taylor series expansion in the neighbourhood of the point $x$, we have

$$
\begin{aligned}
& y^{\prime}(x-\delta) \approx y^{\prime}(x)-\delta y^{\prime \prime}(x) \\
& y^{\prime}(x+\delta) \approx y^{\prime}(x)+\delta y^{\prime \prime}(x)
\end{aligned}
$$

Using Eq. (3)

into Eq. (1), we obtain an asymptotically equivalent singularly perturbed first order differential-difference equation of the form: 


$$
\begin{aligned}
& -\varepsilon y^{\prime \prime}(x)+f(x) y^{\prime}(x)+g(x) y(x)=0 \\
& \text { Where } f(x)=\frac{a(x)}{\tau a(x)-1}, g(x)=\frac{b(x)}{\tau a(x)-1}
\end{aligned}
$$

Transition from Eq. (1) to Eq. (4) is admitted, because of the condition that $0<\varepsilon<<1$ is sufficiently small. This replacement is significant from the computational point of view. Further details on the validity of this transition can be found in Elsgolt's and Norkin [5]. Thus, the solution of Eq. (4) will provide a good approximation to the solution of Eq. (1).

3. Liouville Green Transformation:

Rewrite the equation (4) as below:

$-\varepsilon y^{\prime \prime}(x)+f(x) y^{\prime}(x)+g(x) y(x)=0, \quad x \in[0,1]$

The Liouville-Green transforms $\mathrm{Z}, \varphi(x), v(z)$

$$
\begin{gathered}
z=\varphi(x)=\frac{1}{\varepsilon} \int f(x) d x \\
\phi(x)=\varphi^{\prime}(x)=\frac{1}{\varepsilon} f(x) \\
v(z)=\phi(x) y(x)
\end{gathered}
$$

According (8), we have

$$
\begin{aligned}
& \frac{d y}{d x}=\frac{1}{\phi(x)} \frac{d v}{d z} z^{\prime}(x)-\frac{\phi^{\prime}(x)}{\phi^{2}(x)} v(z)=\frac{\phi^{\prime}(x)}{\phi(x)} \frac{d v}{d z}-\frac{\phi^{\prime}(x)}{\phi^{2}(x)} v(z) \\
& \frac{d^{2} y}{d x^{2}}=\frac{1}{\phi(x)}\left(\left(\varphi^{2}(x) \frac{d^{2} v}{d z^{2}}+\left(\phi^{\prime \prime}-\frac{2 \varphi^{\prime}(x) \phi^{\prime}(x)}{\phi(x)}\right) \frac{d v}{d z}\right)-\left(\frac{\phi^{\prime \prime}(x)}{\phi(x)}-\frac{2 \varphi^{\prime 2}(x)}{\phi^{2}(x)} v\right)\right)
\end{aligned}
$$

From (5),(9) and (10), we obtain

$-\frac{\varepsilon \varphi^{\prime 2}}{\phi} \frac{d^{2} v}{d z^{2}}+\left(\frac{2 \varepsilon \varphi^{\prime} \phi^{\prime}}{\phi^{2}}-\frac{\varepsilon \varphi^{\prime \prime}(x)}{\phi(x)}+f(x) \frac{\varphi^{\prime}(x)}{\phi(x)}\right) \frac{d v}{d z}+\left(\frac{\varepsilon \phi^{\prime \prime}(x)}{\phi^{2}(x)}-\frac{2 \varepsilon \varphi^{\prime 2}(x)}{\phi^{3}(x)}-f(x) \frac{\phi^{\prime}(x)}{\phi^{2}}+\frac{g(x)}{\phi}\right) v(z)=0$, i.

e.

$\frac{d^{2} v}{d z^{2}}+\frac{1}{\varphi^{\prime 2}}\left(\varphi^{\prime \prime}(x)-\frac{2 \varphi^{\prime} \phi^{\prime}}{\phi}-f(x) \frac{\varphi^{\prime}(x)}{\varepsilon}\right) \frac{d v}{d z}-\frac{1}{\varphi^{\prime 2}}\left(\frac{\phi^{\prime \prime}(x)}{\phi(x)}-\frac{2 \phi^{\prime 2}}{\phi^{2}}-f(x) \frac{\phi^{\prime}(x)}{\varepsilon \phi(x)}+\frac{g(x)}{\varepsilon}\right) v(z)=0$,

From (6), we have

$\frac{d^{2} v}{d z^{2}}-\left(\varepsilon \frac{f^{\prime}(x)}{f^{2}(x)}+1\right) \frac{d v}{d z}-\frac{1}{f^{2}(x)}\left(\varepsilon^{2} \frac{f^{\prime \prime}(x)}{f(x)}-2 \varepsilon^{2} \frac{f^{\prime 2}(x)}{f^{2}(x)}-\varepsilon f^{\prime}(x)+\varepsilon g(x)\right) v(z)=0$,

i.e.

$\frac{d^{2} v}{d z^{2}}-\frac{d v}{d z}=\varepsilon \frac{f^{\prime}(x)}{f^{2}(x)} \frac{d v}{d z}+\varepsilon \frac{1}{f^{2}(x)}\left(\varepsilon \frac{f^{\prime \prime}(x)}{f(x)}-2 \varepsilon \frac{f^{\prime 2}(x)}{f^{2}(x)}-f^{\prime}(x)+g(x)\right) v(z)=\varepsilon M(x) \frac{d v}{d z}+\varepsilon N(x) v(z)$

Where $\quad M(x)=\frac{f^{\prime}(x)}{f^{2}(x)}, N(x, \varepsilon)=\frac{1}{f^{2}(x)}\left(\varepsilon \frac{f^{\prime \prime}(x)}{f(x)}-2 \varepsilon \frac{f^{\prime 2}(x)}{f^{2}(x)}-f^{\prime}(x)+g(x)\right)$.

Since $\varepsilon$ is a small parameter $((0<\varepsilon<<1), \varepsilon M(x)$ and $\varepsilon N(x, \varepsilon)$ are sufficiently small on $[0,1]$. So, as $\varepsilon \rightarrow 0$,the right hand side of equation (11) vanishes. 
Therefore, we have

$$
\frac{d^{2} v}{d z^{2}}-\frac{d v}{d z} \approx 0
$$

Therefore, the approximate solutions $v(z)$ of (12) are

$$
v(z) \approx c_{1}+c_{2} e^{z}
$$

Where $c_{1}, c_{2}$ are two arbitrary constants. From (6)-(8), one has the asymptotic solutions of differential equations

$$
y(x)=\frac{v(z)}{\phi(x)}=\varepsilon \frac{v(z)}{f(x)} \approx \frac{\varepsilon}{f(x)}\left(c_{1}+c_{2} e^{\frac{1}{\varepsilon} \int_{0}^{x} f(s) d s}\right)
$$

Where $c_{1}, c_{2}$ are two arbitrary constants to be determined using boundary conditions.

\section{Numerical Experiments:}

To demonstrate the efficiency of the method, we consider two numerical experiments with left-end boundary layer and two numerical experiments with right-end boundary layer. We compare the results with the exact solution of the problems. Also we have plotted the graphs of the exact and computed solution of the problem for different values of $\varepsilon$ and for different values of $\delta$ of $o(\varepsilon)$ and $O(\varepsilon)$, which are represented by solid and dotted lines respectively. The maximum absolute error for the examples not having the exact solution is calculated using the double mesh principle [3] $E^{N}=\max _{0 \leq i \leq N}\left|y_{i}^{N}-y_{2 i}^{2 N}\right|$.

Example 1. Consider a singularly perturbed delay differential equation with right layer:

$$
\varepsilon y^{\prime \prime}(x)-y^{\prime}(x-\delta)-y(x)=0 ; \quad \mathrm{x} \in[0,1] \text { with } \mathrm{y}(0)=1 \text { and } y(1)=-1 .
$$

The exact solution is given by

$$
y(x)=\frac{\left(\left(1+e^{m_{2}}\right) e^{m_{1} x}-\left(e^{m_{1}}+1\right) e^{m_{2} x}\right)}{\left(e^{m_{2}}-e^{m_{1}}\right)}
$$

Where $m_{1}=(1-\sqrt{1+4(\varepsilon+\delta)}) / 2(\varepsilon+\delta) \quad$ and $\quad m_{2}=(1+\sqrt{1+4(\varepsilon+\delta)}) / 2(\varepsilon+\delta)$.

Example 2. Now we consider an example of variable coefficient singularly perturbed delay differential equation with right layer:

$$
\varepsilon y^{\prime \prime}(x)-e^{x} y^{\prime}(x-\delta)-x y(x)=0, \quad \text { with } \mathrm{y}(0)=1, \mathrm{y}(1)=1
$$

Example 3. Now we consider an example of variable coefficient singularly perturbed delay differential equation with left layer:

$$
\varepsilon y^{\prime \prime}(x)+e^{-0.5 x} y^{\prime}(x-\delta)-y(x)=0 \text { with } \mathrm{y}(0)=1, \mathrm{y}(1)=1
$$

Example 4. Consider an example of singularly perturbed delay differential equation with left layer:

$$
\varepsilon y^{\prime \prime}(x)+y^{\prime}(x-\delta)-y(x)=0 ; \quad \mathrm{x} \in[0,1] \quad \text { with } \quad y(0)=1 \text { and } \mathrm{y}(1)=0 .
$$

The exact solution is given by

$$
y(x)=\frac{\left(\left(1-e^{\mathrm{m}_{2}}\right) e^{m_{1} x}+\left(e^{m_{1}}-1\right) e^{m_{2} x}\right)}{\left(e^{m_{1}}-e^{m_{2}}\right)}
$$

where $\quad m_{1}=(-1-\sqrt{1+4(\varepsilon-\delta)}) / 2(\varepsilon-\delta) \quad$ and $m_{2}=(-1+\sqrt{1+4(\varepsilon-\delta)}) / 2(\varepsilon-\delta)$.

\section{Discussions and Conclusions}

This paper envisages the use of Liouville Green Transformation to find the solution of singularly perturbed delay differential equations. First, using Taylor series, the given singularly perturbed delay differential difference equation is approximated by an asymptotically equivalent singularly perturbation problem. Then the Liouville Green 
Transformation is applied to get the solution. The method is demonstrated by implementing several model examples by taking various values for the delay parameter and perturbation parameter.

This method is very easy to implement. The effect of small shifts on the boundary layer solution of the problem has been given by considering several numerical experiments. It is observed that if $\delta=o(\varepsilon)$ and as $\delta$ increases, the thickness of the boundary layer decreases in the case when the solution exhibits layer behaviour on the left side, while in the case of the right side boundary layer, it increases and maximum error decreases as the grid size $h$ decreases in both cases which shows the convergence to the computed solution. This method also gives good results for $\delta=O(\varepsilon)$.

Table 1.

The maximum error of example 1 with $\varepsilon=0.1$ for different values of $\delta$ and grid size $\delta \quad \mathrm{N}$

$10^{2} \quad 10^{3} \quad 10^{4} \quad 10^{5}$

\begin{tabular}{lcccc}
\hline Proposed method & & & & \\
0.01 & $3.1086 \mathrm{e}-012$ & $9.9654 \mathrm{e}-013$ & $1.4899 \mathrm{e}-013$ & $7.6974 \mathrm{e}-014$ \\
0.03 & $1.5543 \mathrm{e}-012$ & $8.4666 \mathrm{e}-013$ & $1.2590 \mathrm{e}-013$ & $6.5157 \mathrm{e}-014$ \\
0.06 & $1.4433 \mathrm{e}-012$ & $6.8956 \mathrm{e}-013$ & $1.0125 \mathrm{e}-013$ & $5.3025 \mathrm{e}-014$ \\
0.08 & $1.8874 \mathrm{e}-012$ & $6.1551 \mathrm{e}-013$ & $9.1149 \mathrm{e}-014$ & $4.7220 \mathrm{e}-014$ \\
Results in [11] & & \multicolumn{4}{c}{} \\
0.01 & 0.01172504 & 0.001225627 & $1.2310781 \mathrm{e}-004$ & $1.2280756 \mathrm{e}-005$ \\
0.03 & 0.01505997 & 0.00158944 & $1.5984072 \mathrm{e}-004$ & $1.5998887 \mathrm{e}-005$ \\
0.06 & 0.02575368 & 0.00281263 & $2.8397624 \mathrm{e}-004$ & $2.8449687 \mathrm{e}-005$ \\
0.08 & 0.04781066 & 0.00562948 & $5.7357242 \mathrm{e}-004$ & $5.7485293 \mathrm{e}-005$
\end{tabular}

Table 2

The maximum error of example 1 with $\varepsilon=0.01$ for different values of $\delta$ and grid size

\begin{tabular}{lcccc}
$\delta$ & $\mathrm{N}$ & & \\
\cline { 2 - 5 } & \multicolumn{5}{c}{$10^{2}$} & $10^{3}$ & $10^{4}$ & $10^{5}$ \\
\hline \multicolumn{2}{l}{ Proposed method } & & & \\
0.001 & $1.1574 \mathrm{e}-011$ & $9.1869 \mathrm{e}-012$ & $1.4510 \mathrm{e}-012$ & $7.6905 \mathrm{e}-013$ \\
0.003 & $6.1062 \mathrm{e}-011$ & $7.8688 \mathrm{e}-012$ & $1.2311 \mathrm{e}-012$ & $6.5084 \mathrm{e}-013$ \\
0.006 & $7.5079 \mathrm{e}-011$ & $6.5016 \mathrm{e}-012$ & $1.0041 \mathrm{e}-012$ & $5.2887 \mathrm{e}-013$ \\
0.008 & $8.0491 \mathrm{e}-011$ & $5.8206 \mathrm{e}-012$ & $8.9428 \mathrm{e}-013$ & $4.7015 \mathrm{e}-013$ \\
Results in $[11]$ & & & 0.012 & \\
0.001 & 0.09073569 & 0.01228700 & 0.00127926 & $1.28459 \mathrm{e}-004$ \\
0.003 & 0.10803507 & 0.01562216 & 0.00164450 & $1.65330 \mathrm{e}-004$ \\
0.006 & 0.12777968 & 0.02630926 & 0.00287019 & $2.89704 \mathrm{e}-004$ \\
0.008 & 0.10040449 & 0.04833890 & 0.00568876 & $5.79477 \mathrm{e}-004$
\end{tabular}

Table 3.

The maximum error of example 2 with $\varepsilon=0.1$ for different values of $\delta$ and grid size

\begin{tabular}{ccc}
$\delta$ & $\mathrm{N}$ & \\
$10^{2}$ & $10^{3}$ & $10^{4}$ \\
\hline
\end{tabular}


Proposed method

$\begin{array}{llll}0.01 & 1.7764 \mathrm{e}-012 & 6.2761 \mathrm{e}-013 & 1.0325 \mathrm{e}-013 \\ 0.03 & 1.3323 \mathrm{e}-012 & 3.6593 \mathrm{e}-013 & 5.6510 \mathrm{e}-014 \\ 0.06 & 1.2212 \mathrm{e}-012 & 2.0173 \mathrm{e}-013 & 3.0531 \mathrm{e}-014 \\ 0.08 & 2.2649 \mathrm{e}-012 & 1.4855 \mathrm{e}-013 & 3.3307 \mathrm{e}-014\end{array}$

Results in [11]

\begin{tabular}{lccc}
0.01 & 0.00632996 & 0.000674268 & $6.7871251 \mathrm{e}-005$ \\
0.03 & 0.00815917 & 0.000882563 & $8.8986856 \mathrm{e}-005$ \\
0.06 & 0.01384760 & 0.001579726 & 0.00016020004 \\
0.08 & 0.02477158 & 0.003173235 & 0.00032602775 \\
\hline
\end{tabular}

Table 4

The maximum error of example 2 with $\varepsilon=0.01$ for different values of $\delta$ and grid size

\begin{tabular}{|c|c|c|c|c|}
\hline \multirow[t]{2}{*}{$\delta$} & \multicolumn{4}{|l|}{$\mathrm{N}$} \\
\hline & $10^{2}$ & 1 & & $10^{4}$ \\
\hline \multicolumn{5}{|c|}{ Proposed method } \\
\hline 0.001 & $2.2204 \mathrm{e}-012$ & $5.4035 \mathrm{e}-013$ & $1.0515 \mathrm{e}-013$ & $5.1559 \mathrm{e}-014$ \\
\hline 0.003 & $2.2204 \mathrm{e}-012$ & $3.4365 \mathrm{e}-013$ & $5.7976 \mathrm{e}-014$ & $3.0543 \mathrm{e}-014$ \\
\hline 0.006 & $2.2204 \mathrm{e}-012$ & $2.0103 \mathrm{e}-013$ & $3.2230 \mathrm{e}-014$ & $3.0543 e-015$ \\
\hline 0.008 & $4.4409 \mathrm{e}-012$ & $1.5116 \mathrm{e}-013$ & $2.4403 \mathrm{e}-014$ & $1.7102 \mathrm{e}-015$ \\
\hline
\end{tabular}

Table 5

The maximum error of example 3 with $\varepsilon=0.1$ for different values of $\delta$ and grid size

\begin{tabular}{lcccc}
$\delta$ & \multicolumn{1}{c}{$\mathrm{N}$} & & \\
& \multicolumn{5}{c}{$10^{3}$} & $10^{4}$ & $10^{5}$ \\
\cline { 2 - 5 } & $10^{2}$ & & & \\
\hline Proposed method & & & \\
0.01 & $2.1094 \mathrm{e}-013$ & $2.9421 \mathrm{e}-014$ & $1.3767 \mathrm{e}-014$ & $2.2498 \mathrm{e}-015$ \\
0.03 & $7.7716 \mathrm{e}-013$ & $3.3751 \mathrm{e}-014$ & $8.3267 \mathrm{e}-015$ & $2.5868 \mathrm{e}-015$ \\
0.06 & $4.4409 \mathrm{e}-013$ & $4.3632 \mathrm{e}-014$ & $6.7724 \mathrm{e}-015$ & $3.3277 \mathrm{e}-015$ \\
0.08 & $4.4409 \mathrm{e}-013$ & $5.3846 \mathrm{e}-014$ & $8.3267 \mathrm{e}-015$ & $4.1120 \mathrm{e}-015$ \\
Result in [11] & & & & \\
0.01 & 0.02281050 & 0.00236357 & $2.37223 \mathrm{e}-004$ & $2.37568 \mathrm{e}-005$ \\
0.03 & 0.01954096 & 0.00201453 & $2.02085 \mathrm{e}-004$ & $2.02390 \mathrm{e}-005$ \\
0.06 & 0.01609366 & 0.00165114 & $1.65546 \mathrm{e}-004$ & $1.65808 \mathrm{e}-005$ \\
0.08 & 0.01439633 & 0.00147352 & $1.47701 \mathrm{e}-004$ & $1.48180 \mathrm{e}-005$
\end{tabular}

Table 6

The maximum error of example 3 with $\varepsilon=0.01$ for different values of $\delta$ and grid size $\delta$

$\mathrm{N}$ 


\begin{tabular}{lcccc}
\multicolumn{1}{c}{$10^{2}$} & $10^{3}$ & $10^{4}$ & $10^{5}$ \\
\hline \multicolumn{2}{c}{ Proposed method } & & & \\
0.001 & $3.3307 \mathrm{e}-013$ & $2.9421 \mathrm{e}-014$ & $2.4425 \mathrm{e}-014$ & $2.2536 \mathrm{e}-015$ \\
0.003 & $3.3307 \mathrm{e}-013$ & $3.3751 \mathrm{e}-014$ & $8.1046 \mathrm{e}-015$ & $2.5876 \mathrm{e}-015$ \\
0.006 & $5.5511 \mathrm{e}-013$ & $4.3521 \mathrm{e}-014$ & $6.7724 \mathrm{e}-015$ & $3.3278 \mathrm{e}-015$ \\
0.008 & $5.5511 \mathrm{e}-013$ & $5.3735 \mathrm{e}-014$ & $8.4377 \mathrm{e}-015$ & $4.1122 \mathrm{e}-015$ \\
Result in [11] & & & & \\
0.001 & 0.16595983 & 0.02210942 & 0.002285665 & $2.29353 \mathrm{e}-004$ \\
0.003 & 0.10803507 & 0.01562216 & 0.00164450 & $1.94192 \mathrm{e}-004$ \\
0.006 & 0.12777968 & 0.02630926 & 0.00287019 & $2.89704 \mathrm{e}-004$ \\
0.008 & 0.10040449 & 0.04833890 & 0.00568876 & $5.79477 \mathrm{e}-004$
\end{tabular}

Table 7

The maximum error of example 4 with $\varepsilon=0.1$ for different values of $\delta$ and grid size

\begin{tabular}{|c|c|c|c|}
\hline \multirow[t]{2}{*}{$\delta$} & \multicolumn{3}{|l|}{$\mathrm{N}$} \\
\hline & $10^{2}$ & $10^{3}$ & $10^{4}$ \\
\hline \multicolumn{4}{|c|}{ Proposed method } \\
\hline 0.01 & $1.6653 e-015$ & $2.2204 \mathrm{e}-016$ & $2.2204 \mathrm{e}-016$ \\
\hline 0.03 & $1.6653 e-015$ & $2.2204 \mathrm{e}-016$ & $2.2204 \mathrm{e}-016$ \\
\hline 0.06 & $1.1102 \mathrm{e}-015$ & $2.2204 \mathrm{e}-016$ & $2.2204 \mathrm{e}-016$ \\
\hline 0.08 & $2.7756 \mathrm{e}-016$ & $2.2204 \mathrm{e}-016$ & $2.2204 \mathrm{e}-016$ \\
\hline \multicolumn{4}{|c|}{ Results in [11] } \\
\hline 0.01 & 0.00575975 & 0.00050842 & $5.02478 \mathrm{e}-005$ \\
\hline 0.03 & 0.003932768 & 0.00036132 & $3.58384 \mathrm{e}-005$ \\
\hline 0.06 & 0.002702569 & 0.00025507 & $2.53643 e-005$ \\
\hline 0.08 & 0.00224689 & 0.00021413 & $2.13134 \mathrm{e}-005$ \\
\hline
\end{tabular}

\section{Table 8}

The maximum error of example 4 with $\varepsilon=0.01$ for different values of $\delta$ and grid size

\begin{tabular}{llll}
$\delta$ & $\mathrm{N}$ & & \\
\\
\hline $10^{2}$ & $10^{3}$ & $10^{4}$ & $10^{5}$
\end{tabular}

Proposed method

$\begin{array}{ccccc}0.001 & 1.3878 \mathrm{e}-015 & 3.6653 \mathrm{e}-016 & 2.0955 \mathrm{e}-016 & 2.0955 \mathrm{e}-016 \\ 0.003 & 1.0408 \mathrm{e}-015 & 2.2204 \mathrm{e}-016 & 1.3878 \mathrm{e}-016 & 1.3878 \mathrm{e}-016 \\ 0.006 & 5.4210 \mathrm{e}-016 & 1.3878 \mathrm{e}-016 & 1.3878 \mathrm{e}-016 & 1.3878 \mathrm{e}-016 \\ 0.008 & 5.8234 \mathrm{e}-017 & 4.8572 \mathrm{e}-017 & 4.8572 \mathrm{e}-017 & 4.8572 \mathrm{e}-017\end{array}$




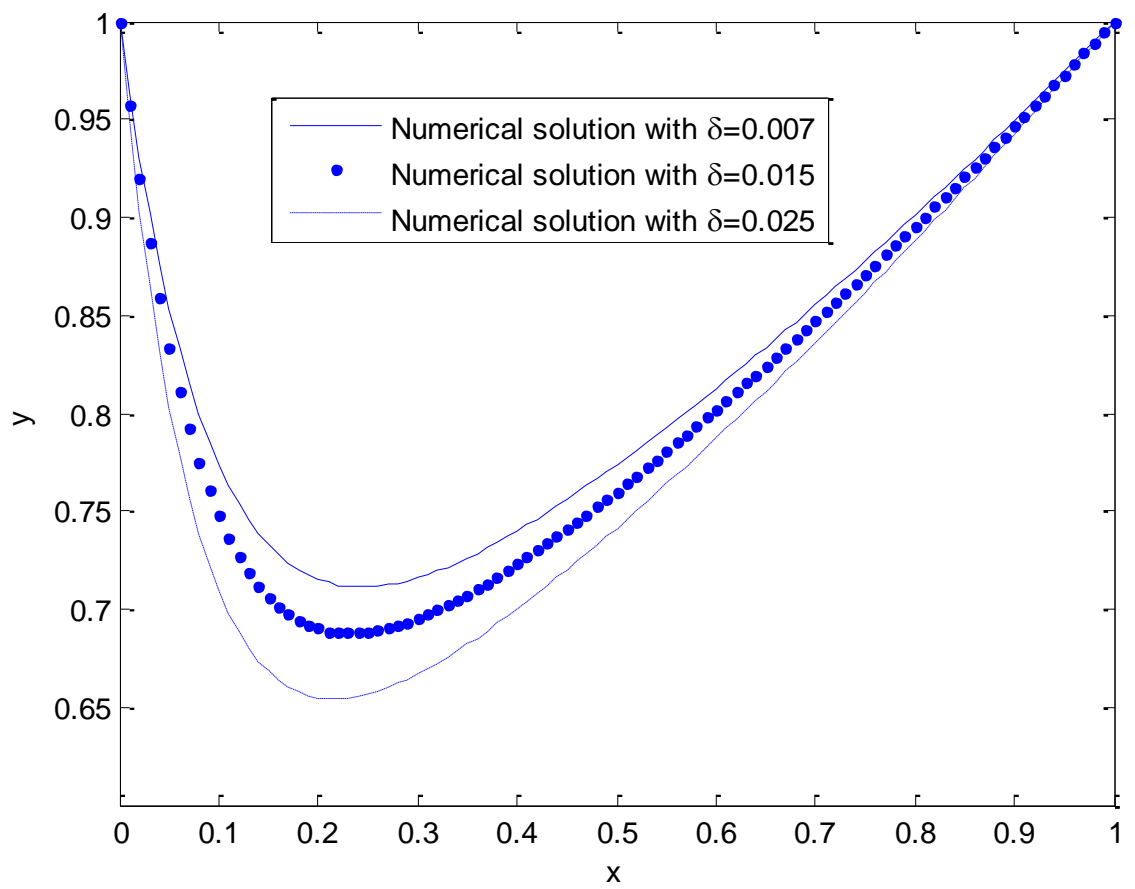

Fig. 1. Example 1 (left 1) for $\varepsilon=0.1$ and for different $\delta$ of o( $\varepsilon$ )

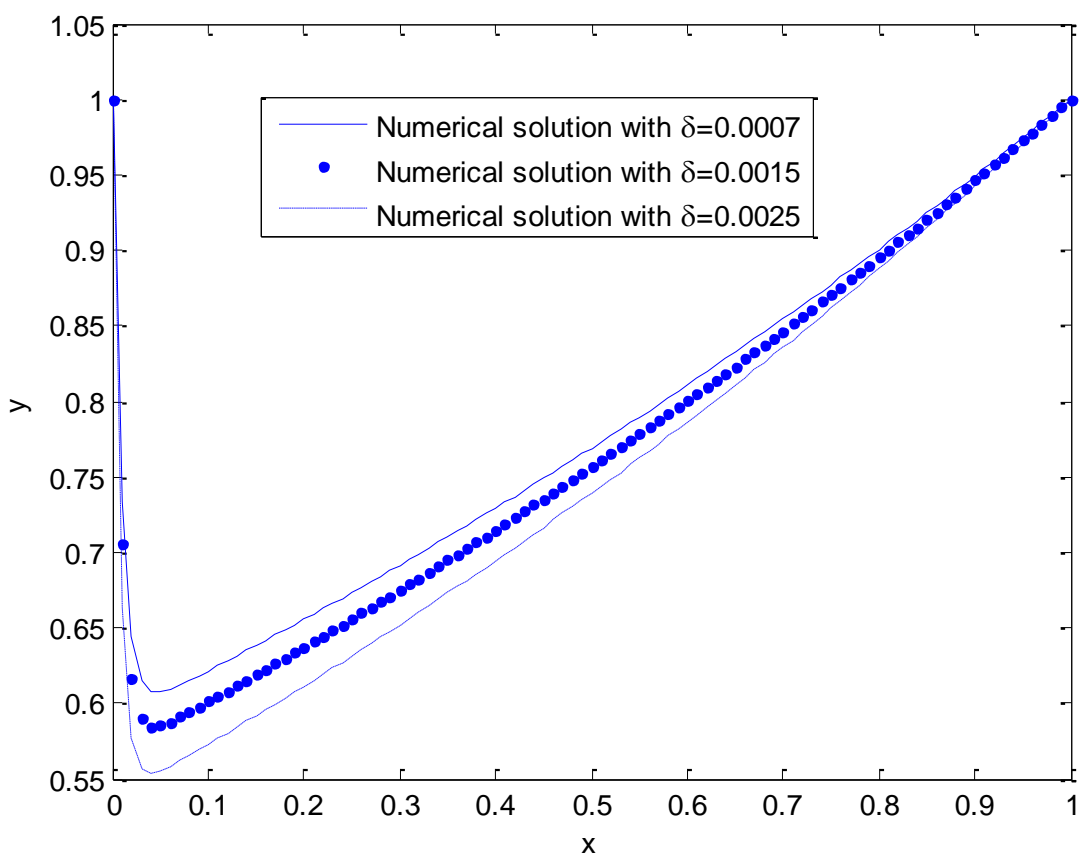

Fig. 2. Example 1 (left 1 ) for $\varepsilon=0.01$ and for different $\delta$ of o $(\varepsilon)$ 


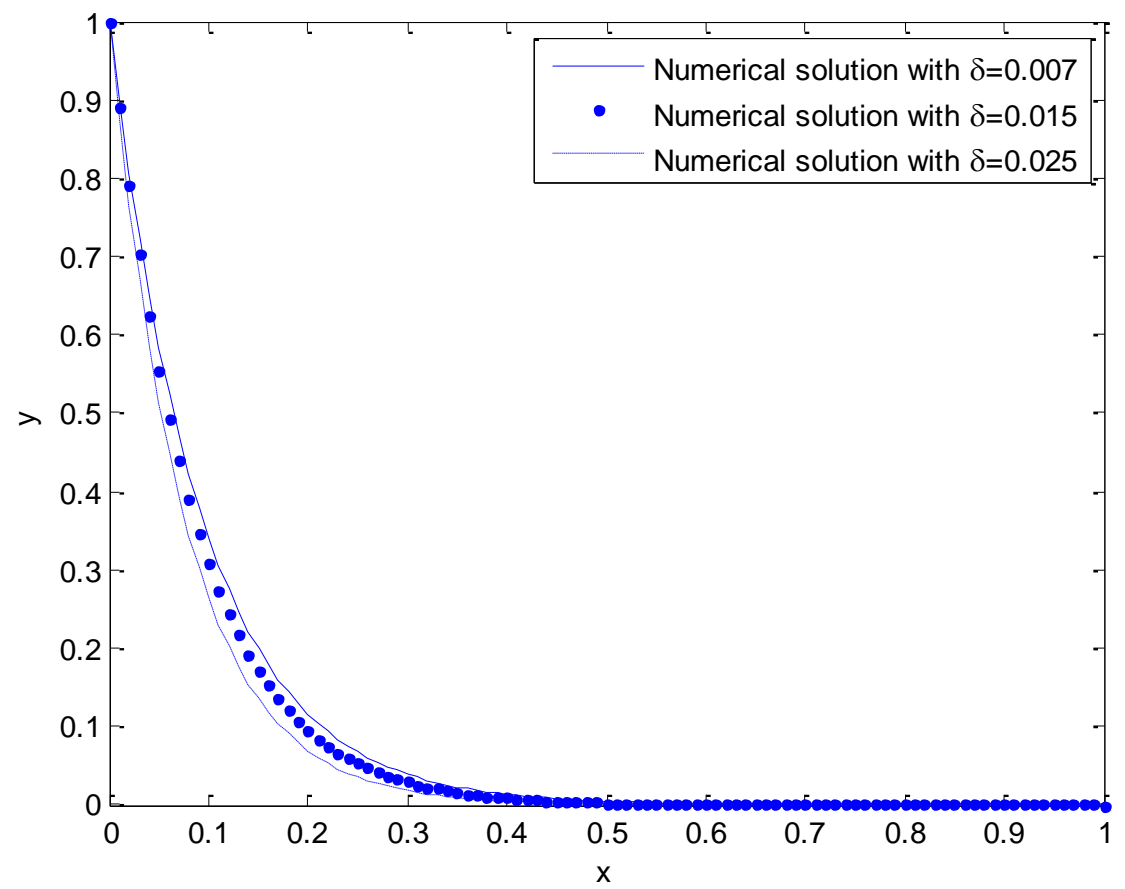

Fig. 3. Example (2) (left 2) for $\varepsilon=0.01$ and for different $\delta$ of $\mathrm{O}(\varepsilon)$

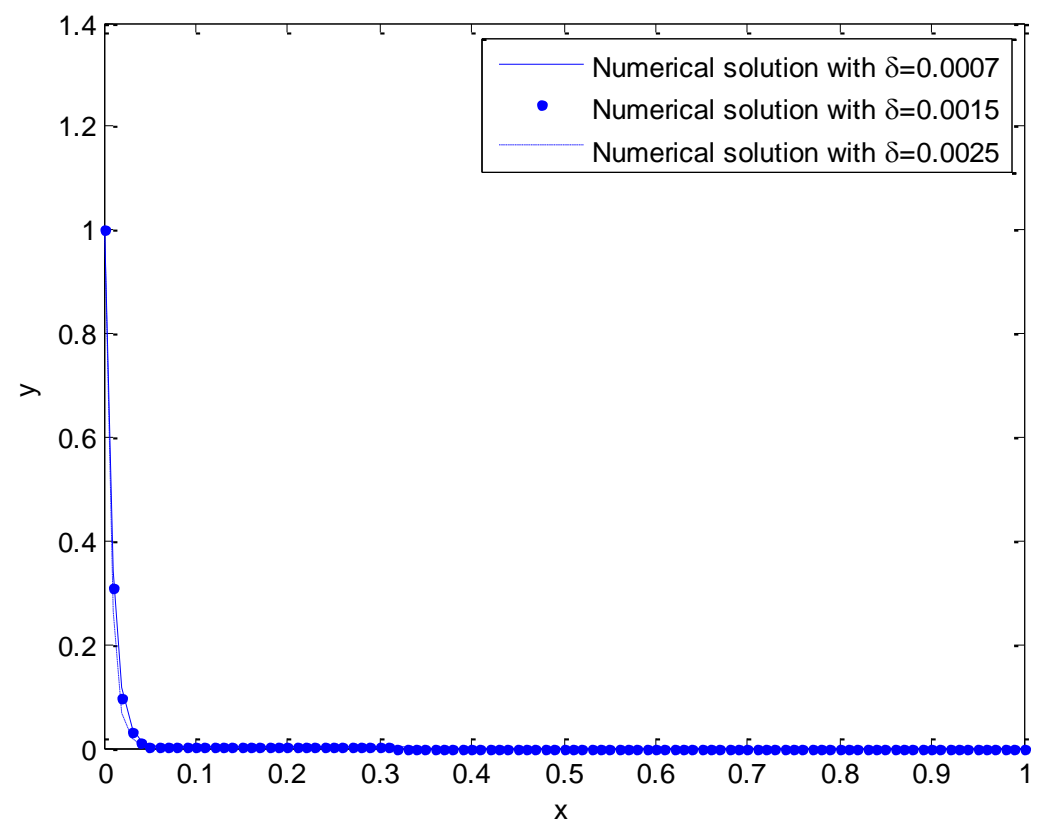

Fig. 4. Example (2) (left 2) for $\varepsilon=0.1$ and for different $\delta$ of o $(\varepsilon)$ 


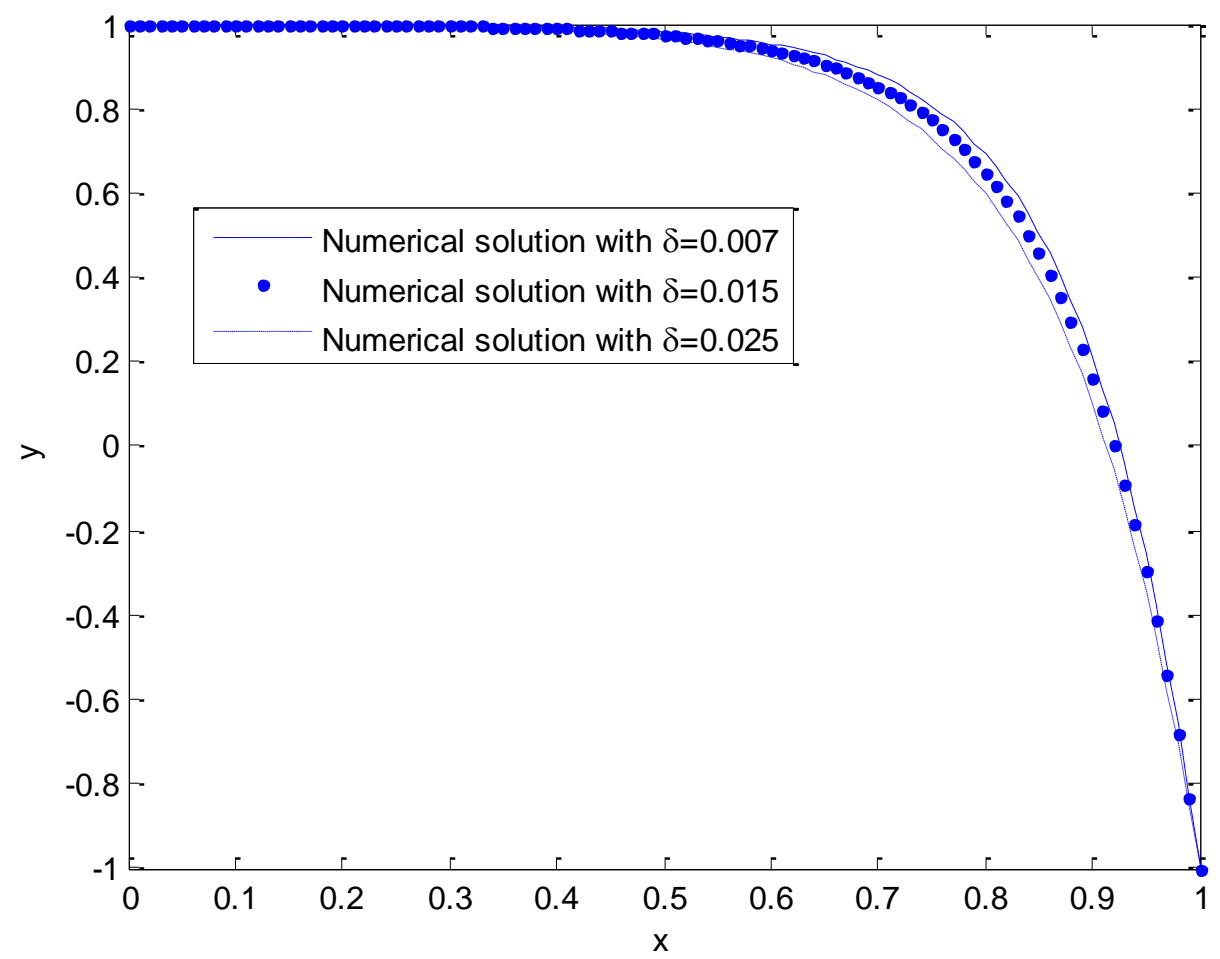

Fig. 5. Example (3) (right 1) for $\varepsilon=0.01$ and for different $\delta$ of o( $\varepsilon$ )

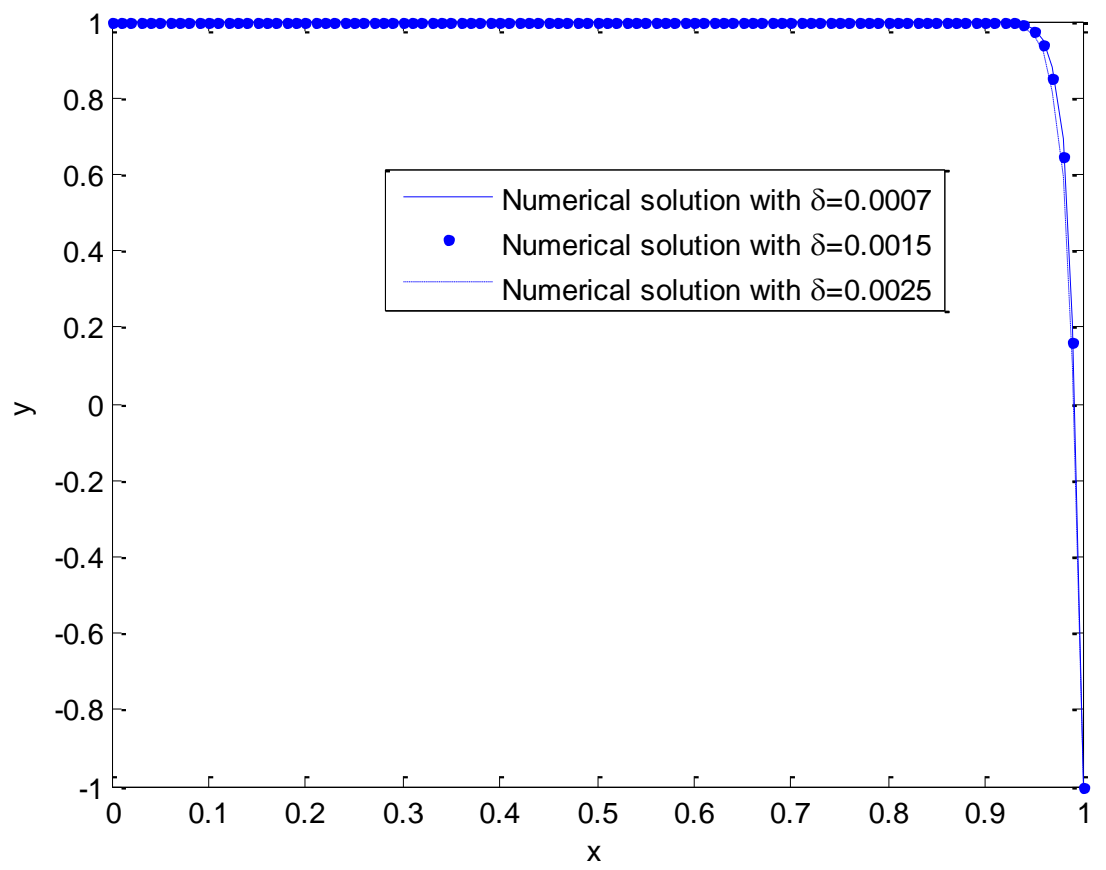

Fig. 6. Example (3) (right 1) for $\varepsilon=0.1$ and for different $\delta$ of o( $\varepsilon$ ) 


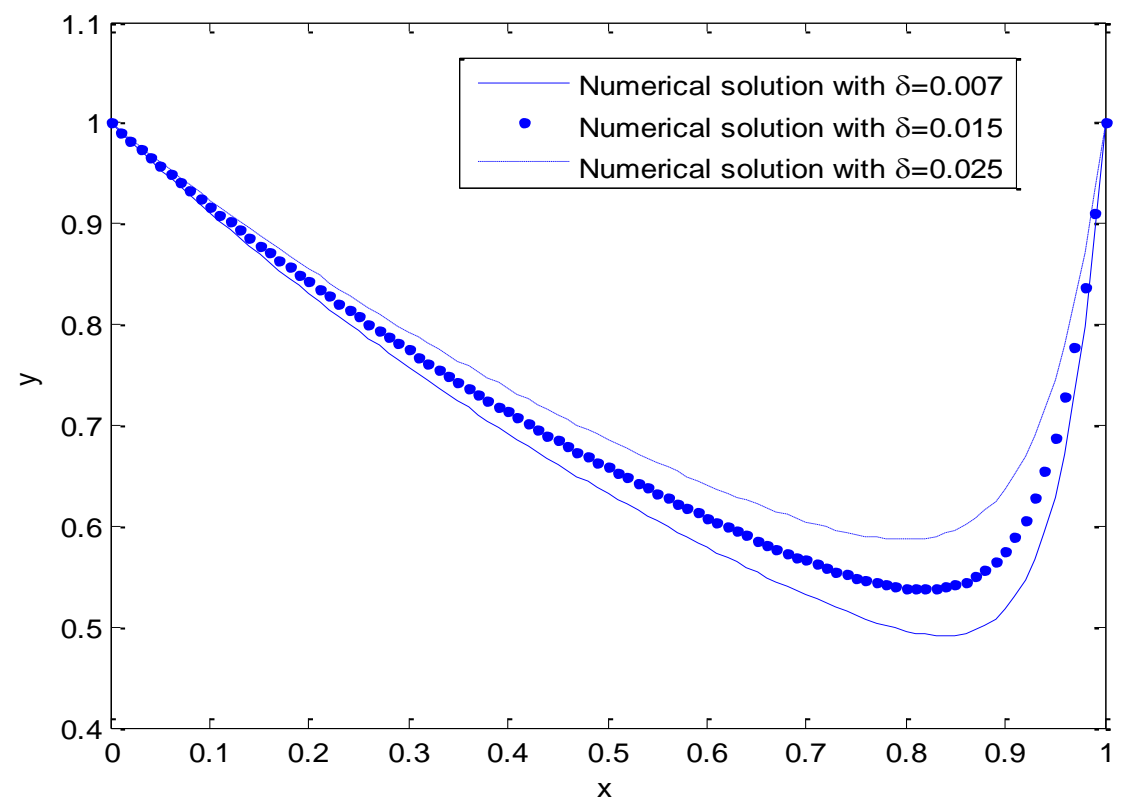

Fig. 7. Example (4) (right 2) for $\varepsilon=0.01$ and for different $\delta$ of $\mathrm{o}(\varepsilon)$

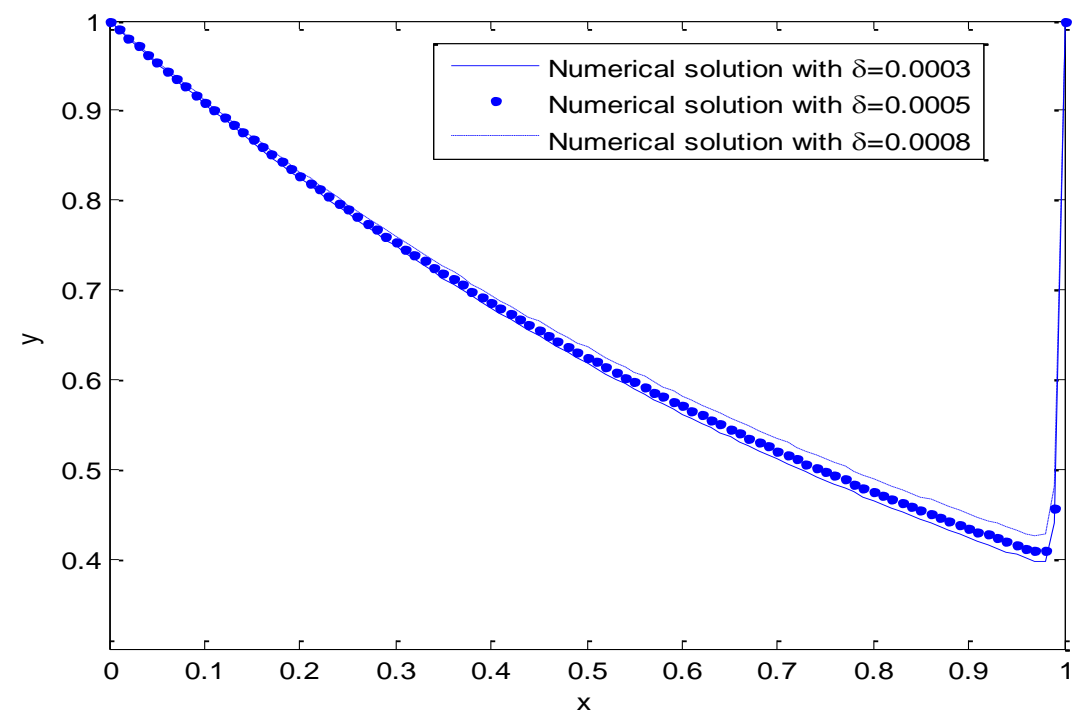

Fig. 8. Example (4) (right 2) for $\varepsilon=0.01$ and for different $\delta$ of $\mathrm{O}(\varepsilon)$

\section{References}

1. Angel, E. and Bellman, R. Dynamic Programming and Partial differential equations, Academic Press, New York, 1972.

2. Derstine, M.W., Gibbs, F.A.H.H.M. and Kaplan, D.L., Bifurcation gap in a hybrid optical system, Phys. Rev. A 26 (1982) 3720-3722.

3. Doolan, E. P., Miller, J. J. H. and Schilders, W. H. A., Uniform Numerical Methods for problems with Initial and Boundary Layers, Boole Press, Dublin, 1980.

4. Elsgolt's, L. E. and Norkin, S. B., Introduction to the Theory and Applications of Differential Equations with Deviating Arguments, Academic Press, New York, 1973. 
5. Glizer, V.Y., Asymptotic analysis and solution of a finite-horizon H1 control problem for singularlyperturbed linear systems with small state delay, J. Optim. Theory Appl. 117 (2003) 295-325.

6. Kadalbajoo, M.K. and Reddy, Y.N., A non asymptotic method for general linear singular perturbation problems, J. Optim. Theory Appl, 55 (1986) 256-269.

7. Kadalbajoo, M.K. and Sharma, K.K., Numerical analysis of singularly perturbed delay differential equations with layer behavior, Applied Mathematics and Computation, 157 (2004) 11-28.

8. Kadalbajoo, M.K. and Sharma, K.K., A numerical method on finite difference for boundary value problems for singularly perturbed delay differential equations, Applied Mathematics and Computation, 197 (2008) 692-707.

9. Lange, C.G. and Miura, R.M., Singular perturbation analysis of boundary-value problems for differentialdifference equations. v. small shifts with layer behavior, SIAM J. Appl. Math. 54 (1994) 249-272.

10. Lange, C.G. and Miura, R.M., Singular perturbation analysis of boundary-value problems for differentialdifference equations. vi. Small shifts with rapid oscillations, SIAM J. Appl. Math. 54 (1994) 273-283.

11. Reddy, Y. N., Soujanya, GBSL, Phaneendra, K., Numerical Integration Method for Singularly Perturbed Delay Differential Equations, International Journal of Applied Science and Engineering,. 10 (3) (2012) 249-261.

12. Stein, R. B.: A theoretical analysis of neuronal variability, Biophys. J., 5 (1965) 173-194. 